\title{
Verschleiß und Verschleißschutz in der Anlagentechnik - Lösungen zur Steigerung der Anlagenverfügbarkeit
}

\author{
Ewald Badisch
}

AC2T research $\mathrm{GmbH}$, Österreichisches Kompetenzzentrum für Tribologie, Wiener Neustadt, Österreich

Online publiziert 25. April 2018

Durch den hohen Wettbewerbsdruck für die österreichische produzierende Industrie ist die Standortsicherung auf Basis technologischen Vorsprungs ein vorrangiges Ziel. Die Anforderungen hinsichtlich Zuverlässigkeit und Standfestigkeit an Prozesse, Anlagen und deren Komponenten steigen und erfordern entsprechende innovative Lösungen.

Im Rahmen dieser Sonderausgabe werden neuartige Lösungsansätze zur Steigerung der Anlagenverfügbarkeit in Stahl-, Zement-, Papier-, Verschleißschutz-Industrie, dem Industrieofenbau sowie der Landtechnik präsentiert. Diese wissenschaftlichen Zugänge reichen dabei von wissensbasierter Werkstoffauswahl auf Basis von Laborexperimenten über Simulation bis hin zu intelligenten Sensoranwendungen zur Zustandsüberwachung. Kostenvorteile in der Produktionstechnik werden erzielt durch eine optimierte Lebensdauer von Verschleiß- und Funktionsteilen in Einklang mit Instandhaltungsintervallen und reduzierten Wartungsaufwänden durch „selbstreinigende Oberflächen“.

\section{Das Österreichische Kompetenzzentrum für Tribologie (AC2T research $\mathrm{GmbH}$ ) als Forschungs- und Entwicklungspartner für die österreichische produzierende Industrie}

Für die Entwicklung und Optimierung von Komponenten, welche höchsten Temperaturen, aggressiven Medien und dadurch auch extremen Verschleiß ausgesetzt sind, bietet $A C^{2} T$ seinen Kunden eine umfassende Forschungsund Entwicklungsumgebung: beginnend bei Hochtemperatur-Analysemöglichkeiten, mit denen der Verschleiß bei Betriebsbedingungen im Labor nachgestellt und verifiziert wird, über Vorort-Messungen bis hin zu Simulationsmodellen für Konstruktion und Optimierung der Komponenten. Mittels dieser High-End F\&E-Infrastruktur und dem lang-

Dipl.-Ing. Dr.mont. E. Badisch ( $\varangle)$

AC2T research $\mathrm{GmbH}$, Österreichisches Kompetenzzentrum für Tribologie,

Viktor-Kaplan-Straße 2/C

2700 Wiener Neustadt, Österreich

ewald.badisch@ac2t.at jährigen Know-How ermöglicht $\mathrm{AC}^{2} \mathrm{~T}$ seinen Kunden, die Zuverlässigkeit und Standfestigkeit ihrer Anlagen und Prozesse zu steigern und somit erhebliche Kosteneinsparung zu realisieren.

Das Thema der fortschreitenden Digitalisierung hat die Anlagentechnik schon längst erreicht und stellt diese vor besondere Herausforderungen. Durch die immer höhere Rechenleistung moderner Computer und die steigende Vernetzung steigen auch die technischen Möglichkeiten: So werden beispielsweise Komponenten oder auch gesamte Prozesse mittels sogenannter Digitaler Zwillinge virtuell nachgebildet. Damit können sowohl das dynamische Systemverhalten wie auch in der Folge das Verschleißverhalten eines neuen Werkstoffes für eine Komponente simuliert und berechnet werden, wodurch eine rasche und effektive Anlagen- und Komponentenauslegung bis hin zu einer Systemoptimierung ermöglicht wird. Zusätzlich werden zukünftig durch die steigende Vernetzung verschiedenste Sensoren gleichzeitig ausgewertet. In Kombination mit intelligenten Auswertealgorithmen, wie z. B. maschinelles Lernen oder künstliche Intelligenz, kann der aktuelle Anlagenzustand berechnet und eine genaue Restlebensdauer in Abhängigkeit der aktuellen Prozessparameter prognostizieren werden. Diese neuen Möglichkeiten werden zukünftig im Baustein für Prädiktive Maintenance in einer vernetzten und digitalen Fabrik nicht mehr wegzudenken sein.

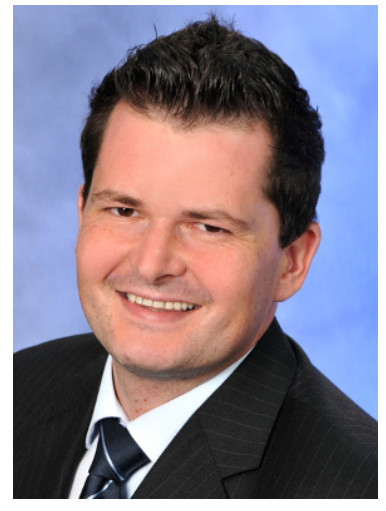

Ewald Badisch 